\title{
On the Generic Uniqueness of Pareto-Efficient Solutions of Vector Optimization Problems
}

\author{
Dejin Zhang $\mathbb{D}^{1,2}$ Shuwen Xiang $\mathbb{D}^{1,3}$ Yanlong Yang, ${ }^{1}$ and Xicai Deng ${ }^{4}$ \\ ${ }^{1}$ School of Mathematics and Statistics, Guizhou University, Guiyang, Guizhou 550025, China \\ ${ }^{2}$ School of Mathematical Sciences, Guizhou Normal University, Guiyang, Guizhou 550025, China \\ ${ }^{3}$ College of Mathematics and Information Science, Guiyang University, Guiyang, Guizhou 550005, China \\ ${ }^{4}$ School of Mathematics and Big Date, Guizhou Education University, Guiyang, Guizhou 550018, China \\ Correspondence should be addressed to Shuwen Xiang; shwxiang@vip.163.com
}

Received 10 November 2020; Accepted 24 June 2021; Published 12 July 2021

Academic Editor: Mahmoud Mesbah

Copyright $\odot 2021$ Dejin Zhang et al. This is an open access article distributed under the Creative Commons Attribution License, which permits unrestricted use, distribution, and reproduction in any medium, provided the original work is properly cited.

In this paper, the generic uniqueness of Pareto weakly efficient solutions, especially Pareto-efficient solutions, of vector optimization problems is studied by using the nonlinear and linear scalarization methods, and some further results on the generic uniqueness are proved. These results present that, for most of the vector optimization problems in the sense of the Baire category, the Pareto weakly efficient solution, especially the Pareto-efficient solution, is unique. Furthermore, based on these results, the generic Tykhonov well-posedness of vector optimization problems is given.

\section{Introduction}

In optimization theory, the uniqueness of the solution is critical to the stability and calculation of the solution, but it is difficult to guarantee the uniqueness of solutions, even for the optimal solution of scalar optimization problems. For this reason, some researchers focus on the generic uniqueness problems, that is, when most of the optimization problems (in the sense of Baire category) have a unique solution (see, e.g., [1] and references therein). Kenderov [1] proved the generic uniqueness of the solution for scalar optimization problems. Beer [2] extended the generic uniqueness to constrained optimization problems in the Čech complete space. The generic uniqueness of optimal solutions was proved for some classes of infinite dimensional linear programming problems (see, e.g., $[3,4])$, and some generic uniqueness results were proposed in linear optimization problems (see, e.g., [5, 6]). Ioffe and Lucchetti [7] presented several types of concepts of generic uniqueness and Tykhonov well-posedness for various classes of optimization problems. Based on the method of genericity, the generic uniqueness of solution was generalized to zero-sum games, variational inequalities, equilibrium problems, and so on (see, e.g., [8-15]).

Tykhonov well-posedness plays a very important role in solving optimization problems by the iterative method (see, e.g., [16]). In vector optimization theory, there are a lot of approaches which give rise to various well-posedness notions (see, e.g., [17-25]). The Pareto-efficient solutions of vector optimization problems are based on partial order, so they are not generally unique and even not generically unique (see Example 1). In view of the multiplicity of Paretoefficient solutions, the generalization of Tykhonov wellposedness for the vector case is less developed. There are two interesting problems that deserve attention. (1) Can some results about the generic uniqueness of the solutions in vector optimization problems be given under certain conditions? (2) Based on the generic uniqueness, what can be given about the generic results of Tykhonov well-posedness under given conditions?

This paper studies the two problems above by the scalarization method, mainly by means of the generic uniqueness and pointwise method of vector optimization problems, and presents the generic uniqueness of Pareto weakly efficient solutions, especially Pareto-efficient 
solutions. Based on the generic uniqueness results above, the generic Tykhonov well-posedness of vector optimization problems is given.

The outline of the paper is as follows. In Section 2, the authors introduce some preliminaries, especially review the nonlinear and linear scalarization method. In Section 3, using nonlinear and linear scalarization methods, we present that, in the sense of the Baire category, for most of the vector optimization problems, the Pareto weakly efficient solution, especially the Pareto-efficient solution, is unique. It follows that the vector optimization problem is generic Tykhonov well-posed at the corresponding value. In Section 4, some conclusions are given.

\section{Preliminaries}

Throughout this paper, unless stated otherwise, we always assume that the feasible domain of optimization problem is a metric space, while $B_{r}(x)$ denotes the open ball with center $x$ and radius $r$, and $\mathbb{R}^{n}$ denotes $n$-dimensional Euclidean space. For convenience, we first recall some definitions and conclusions.

Definition 1. Let $X, Y$ be Hausdorff topological spaces and $F: X \longrightarrow 2^{Y}$ be a set-valued mapping.

(i) $F$ is said to be upper semicontinuous at $x \in X$, iff for each open set $G$ in $Y$ with $G^{F(x)}$, there exists an open neighborhood $O(x)$ of $x$ such that $G^{F\left(x^{\prime}\right)}$ for all $x^{\prime} \in O(x)$.

(ii) $F$ is said to be lower semicontinuous at $x \in X$, iff for each open set $G$ in $Y$ with $G \cap F(x) \neq \varnothing$, there exists an open neighborhood $O(x)$ of $x$ such that $G \cap F\left(x^{\prime}\right) \neq \varnothing$ for all $x^{\prime} \in O(x)$.

(iii) $F$ is said to be continuous at $x \in X$, iff $F$ is both upper semicontinuous and lower semicontinuous at $x$.

(iv) $F$ is said to be an usco mapping, iff for each $x \in X$, $F(x)$ is compact and $F$ is upper semicontinuous at $x$.

First of all, consider the following scalar optimization problem:

$$
(X, f): \min _{x \in X} f(x)
$$

where $f: X \longrightarrow \mathbb{R}$.

The definitions of Tykhonov well-posedness and generalized Tykhonov well-posedness of scalar optimization problem are introduced (for more details and results, see $[17,26])$.

Definition 2. Let $X$ be a Hausdorff topological space and $f: X \longrightarrow \mathbb{R}$ be a function.

(i) The optimization problem $(X, f)$ is called Tykhonov well-posed, iff $f$ has a unique optimal solution $\bar{x}$ on $X$, and for any sequence $\left\{x_{n}\right\} \subset X$ with $f\left(x_{n}\right) \longrightarrow \inf _{x \in X} f(x)$, it holds that $x_{n} \longrightarrow \bar{x}$.

(ii) The optimization problem $(X, f)$ is called generalized Tykhonov well-posed, iff the set of solutions of
$(X, f)$ is nonempty, and for every sequence $\left\{x_{n}\right\} \subset X$ with $f\left(x_{n}\right) \longrightarrow \inf _{x \in X} f(x)$, there exists a subsequence $\left\{x_{n_{k}}\right\}$ of $\left\{x_{n}\right\}$ converging to an optimal solution of $(X, f)$.

In what follows, we will investigate the vector optimization problem as below:

$$
V(X, f): \min _{x \in X} f(x),
$$

where $\quad f: X \longrightarrow \mathbb{R}^{n}, \quad f(x)=\left(f_{1}(x), \ldots, f_{n}(x)\right)$, and $f_{i}(x): X \longrightarrow \mathbb{R}, i \in\{1, \ldots, n\}$.

Definition 3. Let $X$ be a Hausdorff topological space, $f: X \longrightarrow \mathbb{R}^{n}$, and $f(X)=\{y: y=f(x), x \in X\}$; then,

(i) $\bar{y}=\left(\bar{y}_{1}, \ldots, \bar{y}_{n}\right) \in f(X)$ is said to be a Pareto weakly efficient point of $f(X)$, iff there exists no $y=\left(y_{1}, \ldots, y_{n}\right) \in f(X)$, such that $y_{i}<\bar{y}_{i}$ for all $i \in\{1, \ldots, n\}$. Denote the set of all Pareto weakly efficient points of $f(X)$ by $\operatorname{Min}_{w}(f(X))$. $\bar{x} \in X$ is called a Pareto weakly efficient solution of $V(X, f)$, iff $f(\bar{x}) \in \operatorname{Min}_{w}(f(X))$; denote the set of all Pareto weakly efficient solutions of $V(X, f)$ by $E_{w}(X, f)$.

(ii) $\bar{y}=\left(\bar{y}_{1}, \ldots, \bar{y}_{n}\right) \in f(X)$ is said to be a Paretoefficient point of $f(X)$, iff there is no $y=\left(y_{1}, \ldots, y_{n}\right) \in f(X)$, such that $y_{i} \leq \bar{y}_{i}$ for all $i \in\{1, \ldots, n\}$, and at least one strict inequality holds. Denote the set of all Pareto-efficient points of $f(X)$ by $\operatorname{Min}(f(X)) . \bar{x} \in X$ is called a Pareto-efficient solution of $V(X, f)$, iff $f(\bar{x}) \in \operatorname{Min}(f(X))$; denote the set of all Pareto-efficient solutions of $V(X, f)$ by $E(X, f)$.

Some results of existence of Pareto-efficient solutions for vector optimization problems are restated as follows (see, e.g., $[27,28])$.

Lemma 1. If $Y \subset \mathbb{R}^{n}$ is nonempty and compact, then $\operatorname{Min}(Y) \neq \varnothing$, and thus $\operatorname{Min}_{w}(Y) \neq \varnothing$.

Lemma 2. If $X$ is nonempty and compact, $f: X \longrightarrow \mathbb{R}^{n}$ is continuous; then, $E(X, f) \neq \varnothing$, and thus $E_{w}(X, f) \neq \varnothing$.

The scalarization method is a powerful tool for studying vector optimization problems. Two cases, nonlinear scalarization and linear scalarization, are considered in this paper. The nonlinear scalarization function plays an important role in vector optimization problems, and it was first proposed by Gerstewitz [29] and further investigated by Luc [30] and Chen et al. [28].

Definition 4. Let $Y$ be a Hausdorff topological linear space, $C$ be a closed convex cone of $Y$, and int $C \neq \varnothing$. For given $e \in \operatorname{int} C$ and $a \in Y$, the nonlinear scalarization function is defined by

$$
\xi_{e a}(y)=\min \{t \in \mathbb{R}: y \in t e+a-C\},
$$

for any $y \in Y$. 
Clearly, for any $y \in Y$, there exists a unique $t \in \mathbb{R}$, such that $\xi_{e a}(y)=t$. In particular, when $Y=\mathbb{R}^{n}, C=\mathbb{R}_{+}^{n}, e=$ $\left(e_{1}, \ldots, e_{n}\right) \in \operatorname{int} \mathbb{R}_{+}^{n}$ and $a=\left(a_{1}, \ldots, a_{n}\right) \in \mathbb{R}^{n}$; for the sake of argument, an equivalent definition of the nonlinear scalarization function is given as follows:

$$
\xi_{e a}(y)=\max \left\{\frac{y_{i}-a_{i}}{e_{i}}: i=1, \ldots, n\right\}, \quad \forall y \in Y .
$$

Specifically, for $0 \in \mathbb{R}^{n}$, denote $\xi_{e 0}:=\xi_{e}$. It is easy to check that $\xi_{e a}$ is continuous and convex. Moreover, $\xi_{e a}$ is strictly monotone, i.e.,

$$
y^{2}>y^{1} \Rightarrow \xi_{e a}\left(y^{2}\right)>\xi_{e a}\left(y^{1}\right), \quad \forall y^{1}, y^{2} \in Y .
$$

The another typical scalarization method is linear scalarization, that is, the weight method. Let

$$
W=\left\{\omega: \omega=\left(\omega_{1}, \ldots, \omega_{n}\right) \in \mathbb{R}_{+}^{n}, \quad \sum_{i=1}^{n} \omega_{i}=1\right\},
$$

where $\omega$ is called a weight vector. For the vector optimization problem (2), consider the following weighted optimization problem:

$$
\min _{x \in X} \sum_{i=1}^{n} \omega_{i} f_{i}(x)
$$

For any $x \in X$, denote $w_{f, \omega}(x):=\sum_{i=1}^{n} \omega_{i} f_{i}(x)$. For $\bar{x} \in X$, if $w_{f, \omega}(\bar{x})=\min _{x \in X} w_{f, \omega}(x)$ holds, then $\bar{x}$ is called a weighted solution of vector optimization problem (2) with respect to $\omega$. Denote

$$
F_{w}(f, \omega):=\left\{\bar{x}: w_{f, \omega}(\bar{x})=\min _{x \in X} w_{f, \omega}(x)\right\},
$$

the set of all weighted solution of the vector optimization problem (2) with respect to $\omega$. In fact, for each $f: X \longrightarrow \mathbb{R}^{n}$ and $w \in W, F_{w}(f, \omega) \subset E_{w}(X, f)$.

\section{Main Results}

In this section, let $X$ be a nonempty compact set, $C=\mathbb{R}_{+}^{n}$, and $e=\left(e_{1}, \ldots, e_{n}\right) \in \operatorname{int} \mathbb{R}_{+}^{n}$. Let

$$
C^{n}(X)=\left\{f=\left(f_{1}, \ldots, f_{n}\right) \mid f_{i}: X \longrightarrow \mathbb{R} \text { is continuous, } \quad \forall i=1, \ldots, n\right\}
$$

The norm on $C^{n}(X)$ is defined by

$$
\|f(x)\|_{\infty}=\max _{x \in X} \max \left\{\left|f_{1}(x)\right|, \ldots,\left|f_{n}(x)\right|\right\} .
$$

It is easy to verify that $\left(C^{n}(X),\|\cdot\|_{\infty}\right)$ is a complete metric space.

For any fixed $e \in \operatorname{int} C, a \in \mathbb{R}^{n}$, and $f \in C^{n}(X)$, define the nonlinear scalarization function $\xi_{e a}^{f}: X \mapsto \mathbb{R}$ :

$$
\xi_{e a}^{f}(x):=\xi_{e a}(f(x)), \quad \forall x \in X .
$$

From the continuity of $f$ and $\xi_{e a}$, we get that $\xi_{e a}^{f}$ is continuous on $X$. Next, we will present some properties of nonlinear scalarization function $\xi_{e a}^{f}$.

Lemma 3. For $(f, a) \in C^{n}(X) \times \mathbb{R}^{n}$,

(i) $\xi_{\text {ea }}^{f}$ has a minimum value on $X$.

(ii) $\bar{x} \in E_{w}(f, X) \Leftrightarrow \xi_{e f(\bar{x})}^{f}(\bar{x})=\min _{x \in X} \xi_{e f(\bar{x})}^{f}(x)=0$.

Proof

(i) By the continuity of $\xi_{e a}^{f}$ and compactness of $X$, it is easy to verify that $\xi_{e a}^{f}$ has a minimum value on $X$.

(ii) “ $\Leftarrow$ " Suppose by contradiction that $\bar{x} \notin E_{w}(f, X)$; then, there exists $x / \in X$, such that $f\left(x^{\prime}\right)<f(\bar{x})$, and by the strict monotony of $\xi_{e a}$, we have

$$
\xi_{e f(\bar{x})}^{f}\left(x^{\prime}\right)=\xi_{e f(\bar{x})}\left(f\left(x^{\prime}\right)\right)<\xi_{e f(\bar{x})}(f(\bar{x}))=\xi_{e f(\bar{x})}^{f}(\bar{x}),
$$

which is in contradiction with the minimal point $\bar{x}$ of $\xi_{e f(\bar{x})}^{f}$ on $X$, and thus $\bar{x} \in E_{w}(f, X)$.

“ $\Rightarrow$ " For every $\bar{x} \in E_{w}(f, X)$, firstly, by the definition of $\xi_{e a}^{f}$ and $\xi_{e a}$, let $a=f(\bar{x})$, and we have $\xi_{e f(\bar{x})}^{f}(\bar{x})=0$. In what follows, we will show that $\xi_{e f(\bar{x})}^{f}(\bar{x})=\min _{x \in X} \xi_{e f(\bar{x})}^{f}(x)$. Suppose by contradiction that there exists $x / \in X$, such that $\xi_{e f(\bar{x})}^{f}(x \prime)=\xi_{e f(\bar{x})}(f(x \prime))<0$; by the definition of $\xi_{e a}$, there exists $t ı<0$ such that $f(x /) \in f(\bar{x})+t / e-C$; hence, $f(x /)-f(\bar{x}) \in-$ int $C$, which is in contradiction with $\bar{x} \in E_{w}(f, X)$.

For every $f \in C^{n}(X)$ and $a \in \mathbb{R}^{n}$, denote $T^{\xi}(f, a):=\xi_{e a}^{f}$; then, the result is as follows.

Lemma 4. $T^{\xi}: C^{n}(X) \times \mathbb{R}^{n} \mapsto C(X)$ is a continuous mapping.

Proof. Assume by contradiction that there exists a sequence $\left\{\left(f^{m}, a^{m}\right)\right\} \subset C^{n}(X) \times \mathbb{R}^{n}$ with $\left(f^{m}, a^{m}\right) \longrightarrow(f, a)$ such that $T^{\xi}\left(f^{m}, a^{m}\right)$ do not converge to $T^{\xi}(f, a)$; it means that there exist $\varepsilon_{0}>0$ and a subsequence of $\left\{\left(f^{m}, a^{m}\right)\right\}$, without loss of generality, still denoted by $\left\{\left(f^{m}, a^{m}\right)\right\}$, and exist corresponding $x_{m} \in X$, such that

$$
\left|\xi_{e a^{m}}^{f^{m}}\left(x_{m}\right)-\xi_{e a}^{f}\left(x_{m}\right)\right|=\left|\xi_{e a^{m}}\left(f^{m}\left(x_{m}\right)\right)-\xi_{e a}\left(f\left(x_{m}\right)\right)\right| \geq \varepsilon_{0} .
$$

Without loss of generality, let $\xi_{e a^{m}}\left(f^{m}\left(x_{m}\right)\right)-$ $\xi_{e a}\left(f\left(x_{m}\right)\right) \geq \varepsilon_{0} ; \quad$ otherwise, consider $\xi_{e a}\left(f\left(x_{m}\right)\right)-$ $\xi_{\text {ea }}\left(f^{m}\left(x_{m}\right)\right) \geq \varepsilon_{0}$. 
By $\xi_{e a^{m}}\left(f^{m}\left(x_{m}\right)\right)-\xi_{e a}\left(f\left(x_{m}\right)\right) \geq \varepsilon_{0}, \quad \xi_{e a^{m}}\left(f^{m}\left(x_{m}\right)\right)-$ $\varepsilon_{0} \geq \xi_{e a}\left(f\left(x_{m}\right)\right)$. From $\xi_{e a}(y)=\max \left\{\left(\left(y_{i}-a_{i}\right) / e_{i}\right)\right.$ : $i=1, \ldots, n\}$, we have

$$
\begin{aligned}
& \max \left\{\frac{f_{i}^{m}\left(x_{m}\right)-a_{i}^{m}}{e_{i}}: i=1, \ldots, n\right\} \\
& -\varepsilon_{0} \geq \max \left\{\frac{f_{i}\left(x_{m}\right)-a_{i}}{e_{i}}: i=1, \ldots, n\right\} .
\end{aligned}
$$

Let $\quad\left(f_{i_{0}}^{m}\left(x_{m}\right)-a_{i_{0}}^{m} / e_{i_{0}}\right)=\max \left\{\left(f_{i}^{m}\left(x_{m}\right)-a_{i}^{m} / e_{i}\right): i=\right.$ $1, \ldots, n\}$; then,

$$
\frac{f_{i_{0}}^{m}\left(x_{m}\right)-a_{i_{0}}^{m}}{e_{i_{0}}}-\varepsilon_{0} \geq \max \left\{\frac{f_{i}\left(x_{m}\right)-a_{i}}{e_{i}}: i=1, \ldots, n\right\} .
$$

Therefore,

$$
\frac{f_{i_{0}}^{m}\left(x_{m}\right)-a_{i_{0}}^{m}}{e_{i_{0}}}-\varepsilon_{0} \geq \frac{f_{i_{0}}\left(x_{m}\right)-a_{i_{0}}}{e_{i_{0}}} .
$$

Hence,

$$
f_{i_{0}}^{m}\left(x_{m}\right)-f_{i_{0}}\left(x_{m}\right) \geq \varepsilon_{0} e_{i_{0}}+a_{i_{0}}^{m}-a_{i_{0}} .
$$

Note that $e_{i_{0}}>0$ and from $a^{m} \longrightarrow a$ that $a_{i_{0}}^{m}-{ }_{a}^{i_{0}}>-(1 / 2) \varepsilon_{0} e_{i_{0}}$ as $m$ is large enough, then

$$
\left\|f-f^{m}\right\|_{\infty} \geq\left|f_{i_{0}}^{m}\left(x_{m}\right)-f_{i_{0}}\left(x_{m}\right)\right| \geq \frac{1}{2} \varepsilon_{0} e_{i_{0}},
$$

as $m$ is large enough, which is in contradiction with $f^{m} \longrightarrow f$.

For each $f \in C^{n}(X)$, let $F(f):=E_{w}(X, f)$. By Lemma 2, $F(f) \neq \varnothing$; therefore, $F: C^{n}(X) \longrightarrow 2^{X}$ is a set-valued mapping with nonempty values. For each fixed $e \in \operatorname{int} C$ and every $(f, a) \in C^{n}(X) \times \mathbb{R}^{n}$, there exists a corresponding nonlinear scalarization function $\xi_{e a}^{f}$. Denote all minimal points of $\xi_{e a}^{f}$ by $M(f, a):=F\left(\xi_{e a}^{f}\right)$; by Lemma 3 (i), $M(f, a) \neq \varnothing$, and thus we define

$$
M: C^{n}(X) \times \mathbb{R}^{n} \longrightarrow 2^{X} .
$$

Moreover, if $a \in E_{w}(X, f)$, then

$$
M(f, a) \subset E_{w}(X, f)=F(f) .
$$

We introduce the Fort theorem and some results of continuity of Pareto-efficient solutions (for more details and results, see $[31,32])$.

Lemma 5. (Fort theorem) Let $X$ be a complete metric space and $T: X \longrightarrow 2^{Y}$ be an usco mapping; then, there exists a dense residual subset $Q$ of $X$, such that $T$ is continuous at each $x \in Q$.

It is easy to see that the following result holds (see [32]).

Lemma 6. $F: C^{n}(X) \longrightarrow 2^{X}$ is an usco mapping.
Remark 1. From Fort theorem and Lemma 6, it is easy to observe that the usco mapping $F$ is generic continuous on $X$; in other words, it is continuous at most of the points of $X$ in the sense of Baire category.

Lemma 7. For each fixed point $e \in \operatorname{int} C, M: C^{n}(X) \times$ $\mathbb{R}^{n} \longrightarrow 2^{X}$ is an usco mapping.

Proof. By the definition of $M, F$, and $T^{\xi}$, for $(f, a) \in C^{n}(X) \times \mathbb{R}^{n}$, we have

$$
M(f, a)=F\left(\xi_{\text {ea }}^{f}\right)=F\left(T^{\xi}(f, a)\right) .
$$

Since $F$ is usco on $C(X)$ and $T^{\xi}$ is continuous on $C^{n}(X) \times \mathbb{R}^{n}$, we get that $M$ is usco on $C^{n}(X) \times \mathbb{R}^{n}$.

We introduce the following results (see Theorem 3.1 of $[32])$.

Proposition 1. Let $x^{*} \in E(X, f)$ be an Pareto-efficient solution of $f$. If there is some $w \in \mathbb{R}_{+}^{n}$ such that $x^{*}$ is the unique weighted solution of problem (7) with weight vector $\omega$, then $x^{*}$ is essential, that is, for given $\varepsilon>0$, there exist some $\delta>0$ such that $E(X, g) \cap B_{\varepsilon}\left(x^{*}\right) \neq \varnothing$ whenever $\|g-f\|_{\infty}<\delta$.

The following example shows that Pareto-efficient solutions of vector optimization problems do not have the generic uniqueness in general; that is, the set $Q$ of functionals for which there is a unique Pareto-efficient solution to problem (2) is not always a dense residual subset of $C^{n}(X)$.

Example 1. Let $X=[-1,1]$ and $f: X \mapsto \mathbb{R}^{2}$ be defined by

$$
f(x)= \begin{cases}(0,-x), & 0 \leq x \leq 1 \\ (x, 0), & -1 \leq x<0 .\end{cases}
$$

Then, $f$ is continuous and quasiconvex on compact convex set $X$ (see Figure 1).

In this case, we have

$$
\begin{aligned}
\operatorname{Min}(f(X)) & =\{(-1,0),(0,-1)\} \\
E(X, f) & =\{-1,1\} .
\end{aligned}
$$

Then, $x=-1$ and $x=1$ are the unique weighted solutions of $f$ subject to weight vectors $(2 / 3,1 / 3)$ and $(1 / 3,2 / 3)$, respectively, so that they are essential. The concept of essential solution implies that for given $\varepsilon_{0}=1 / 3$, there exist some $\delta>0$ such that $E\left(X, f^{\prime}\right) \cap(-4 / 3,-2 / 3) \neq \varnothing$ and $E\left(X, f^{\prime}\right) \cap(2 / 3,4 / 3) \neq \varnothing \quad$ whenever $\|g-f\|_{\infty}<\delta$. Let $x_{1} \in E\left(X, f^{\prime}\right) \cap(-4 / 3,-2 / 3)$ and $x_{2} \in E\left(X, f^{\prime}\right) \cap \quad(2 / 3$, $4 / 3)$. Then, $x_{1} \neq x_{2}$ and $x_{1}, x_{2} \in E\left(X, f^{\prime}\right)$ for all $f^{\prime} \in B_{\delta}(f)$, which suggests that the set of functions for which there is a unique Pareto-efficient solution is not dense in $C^{2}(X)$.

Based on Lemmas 5 and 7, the generic uniqueness result of Pareto weakly efficient solution of vector optimization problems can be obtained as follows.

Theorem 1. There exists a dense residual subset $Q \subset C^{n}(X) \times \mathbb{R}^{n}$, such that for every $(f, a) \in Q$, if $a \in f\left(E_{w}(X, f)\right)$, then the Pareto weakly efficient solution of 


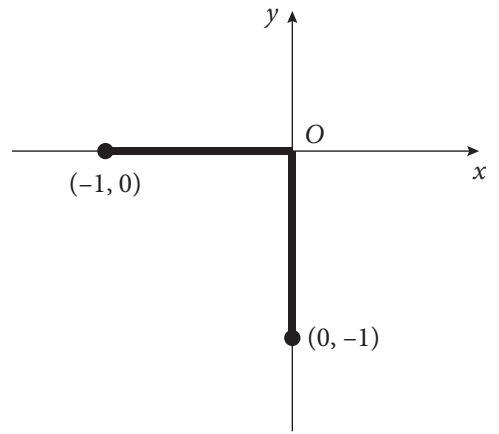

Figure 1: The image of $\mathrm{f}$.

vector optimization problem $V(X, f)$ corresponding to $a$ is unique, that is, $f^{-1}(a)$ is a singleton.

Proof. By Lemmas 5 and 7, for each fixed $e \in \operatorname{int} C$, there exists a dense residual subset $Q \subset C^{n}(X) \times \mathbb{R}^{n}$, such that $M$ is continuous at each $(f, a) \in Q$. In what follows, we will prove that for each $(f, a) \in Q$ and $a \in f\left(E_{w}(X, f)\right), f^{-1}(a)$ is a singleton.

Assume by contradiction that there are $\bar{x}_{1}, \bar{x}_{2} \in$ $E_{w}(X, f)=F(f) \quad$ with $\quad \bar{x}_{1} \neq \bar{x}_{2}$, satisfying $f\left(\bar{x}_{1}\right)=$ $f\left(\bar{x}_{2}\right)=a$.

It follows from $\bar{x}_{1} \neq \bar{x}_{2}$ that there exists $\delta>0$, such that $\overline{B_{\delta}\left(\bar{x}_{1}\right)} \cap \overline{B_{\delta}\left(\bar{x}_{2}\right)}=\varnothing$. Since $\bar{x}_{1}, \bar{x}_{2} \in E_{w}(X, f)$ and by Lemma 3 , we have $\bar{x}_{1}, \bar{x}_{2} \in M(f, a)$.

Define the function $\alpha: X \mapsto \mathbb{R}$ by

$$
\alpha(x)=\frac{\mathrm{d}\left(x, \overline{B_{\delta}\left(\overline{x_{2}}\right)}\right)}{\mathrm{d}\left(x, \overline{B_{\delta}\left(\overline{x_{1}}\right)}\right)+\mathrm{d}\left(x, \overline{B_{\delta}\left(\overline{x_{2}}\right)}\right)}, \quad x \in X .
$$

Let $\left\{\delta_{m}\right\}$ be a sequence of positive numbers satisfying $\delta_{m} \longrightarrow 0$; define the vector-valued functions $f^{m}: X \longrightarrow \mathbb{R}^{n}$ by

$$
f^{m}(x)=f(x)-\alpha(x) \delta_{m} e, \quad x \in X .
$$

Then, $\left\{f^{m}\right\}$ is a sequence of vector-valued functions; obviously, $\left\{f^{m}\right\} \subset C^{n}(X)$. Since

$$
\begin{aligned}
\left\|f^{m}-f\right\|_{\infty} & =\max _{x \in X} \max \left\{\left|f_{1}^{m}(x)-f_{1}(x)\right|, \ldots,\left|f_{n}^{m}(x)-f_{n}(x)\right|\right\}, \\
& =\max _{x \in X} \max \left\{\alpha(x) \delta_{m} e_{1}, \ldots, \alpha(x) \delta_{m} e_{n}\right\} \\
& =\delta_{m} \max \left\{e_{1}, \ldots, e_{n}\right\} \longrightarrow 0,
\end{aligned}
$$

it means that $f^{m} \longrightarrow f$. For every $x_{2} \in B_{\delta}\left(\bar{x}_{2}\right)$, we have

$$
\begin{aligned}
& f^{m}\left(x_{2}\right)=f\left(x_{2}\right)-\alpha\left(x_{2}\right) \delta_{m} e=f\left(x_{2}\right), \\
& f^{m}\left(\bar{x}_{1}\right)=f\left(\bar{x}_{1}\right)-\alpha\left(\bar{x}_{1}\right) \delta_{m} e=f\left(\bar{x}_{1}\right)-\delta_{m} e .
\end{aligned}
$$

In what follows, we only need to show that

$$
x_{2} \notin M\left(f^{m}, a\right) \text {. }
$$

If it is not true, assume that $x_{2} \in M\left(f^{m}, a\right)=F\left(\xi_{e a}^{f^{m}}\right)$; then,

$$
\xi_{e a}\left(f^{m}\left(x_{2}\right)\right)=\min _{x \in X} \xi_{e a}\left(f^{m}(x)\right) \leq \xi_{e a}\left(f^{m}\left(\bar{x}_{1}\right)\right) .
$$

Therefore,

$$
\xi_{e a}\left(f\left(x_{2}\right)\right) \leq \xi_{e a}\left(f\left(\bar{x}_{1}\right)-\alpha\left(\bar{x}_{1}\right) \delta_{m} e\right)=\xi_{e a}\left(f\left(\bar{x}_{1}\right)-\delta_{m} e\right) .
$$

By the definition of $\xi_{e a}\left(f\left(\bar{x}_{1}\right)\right)$, we have

$$
f\left(\bar{x}_{1}\right) \in a+\xi_{e a}\left(f\left(\bar{x}_{1}\right)\right) e-C .
$$

Hence,

$$
f\left(\bar{x}_{1}\right)-\delta_{m} e \in a+\xi_{e a}\left(f\left(\bar{x}_{1}\right)\right) e-\delta_{m} e-C .
$$

That is,

$$
f\left(\bar{x}_{1}\right)-\delta_{m} e \in a+\left[\xi_{e a}\left(f\left(\bar{x}_{1}\right)\right)-\delta_{m}\right] e-C .
$$

Therefore,

$$
\xi_{e a}\left(f\left(\bar{x}_{1}\right)-\delta_{m} e\right) \leq \xi_{e a}\left(f\left(\bar{x}_{1}\right)\right)-\delta_{m} .
$$

From the foregoing, we have

$$
\xi_{e a}\left(f\left(x_{2}\right)\right) \leq \xi_{e a}\left(f\left(\bar{x}_{1}\right)\right)-\delta_{m} .
$$

Hence, $\quad \xi_{e a}\left(f\left(x_{2}\right)\right)<\xi_{e a}\left(f\left(\bar{x}_{1}\right)\right)$, that is, $\xi_{e a}^{f}\left(x_{2}\right)<\xi_{e a}^{f}\left(\bar{x}_{1}\right)$, which is in contradiction with

$$
\xi_{e a}^{f}\left(\bar{x}_{1}\right)=\min _{x \in X} \xi_{e a}^{f}(x) .
$$

Therefore, for every $x_{2} \in B_{\delta}\left(\bar{x}_{2}\right)$, we have $x_{2} \notin M\left(f^{m}, a\right)$, and thus $B_{\delta}\left(\bar{x}_{2}\right) \cap M\left(f^{m}, a\right)=\varnothing$, this is a contradiction with that $M$ is continuous at $(f, a)$.

The following corollary is an immediate consequence of Theorem 1.

Corollary 1. There exists a dense residual subset $Q \subset C^{n}(X) \times \mathbb{R}^{n}$, such that for every $(f, a) \in Q$, if $a \in f(E(X, f))$, then the Pareto-efficient solution of vector optimization problem $V(X, f)$ corresponding to a is unique, that is, $f^{-1}(a)$ is a singleton.

Next, we consider weighted optimization problem (7). For every $f \in C^{n}(X)$ and each $\omega=\left(\omega_{1}, \ldots, \omega_{n}\right) \in W$, it is clear that $F_{w}(f, \omega) \neq \varnothing$, and then $F_{w}: C^{n}(X) \times W \mapsto 2^{X}$ is a set-valued mapping with nonempty values. We recall a result on the continuity of weighted solutions (see, e.g., [32]).

Lemma 8. $F_{w}: C^{n}(X) \times W \longrightarrow 2^{X}$ is an usco mapping. 
It follows from Lemmas 5 and 8 that the generic uniqueness result of Pareto weakly efficient solutions of vector optimization problems can be given as follows.

Theorem 2. There exists a dense residual subset $Q \subset C^{n}(X) \times W$, such that for every $(f, \omega) \in Q$, if $a \in f\left(F_{w}(f, w)\right)$, then the vector a corresponds to the unique Pareto weakly efficient solution of vector optimization problem $V(X, f)$, that is, $f^{-1}(a)$ is a singleton.

Proof. By Lemmas 5 and 8, there exists a dense residual $Q \subset C^{n}(X) \times W$, such that $F_{\omega}$ is continuous at each $(f, \omega) \in Q$. Next, we will prove that for every $(f, \omega) \in Q$, if $a \in f\left(F_{w}(f, \omega)\right)$, then the vector $a$ corresponds to a unique Pareto weakly efficient solution of vector optimization problem (7).

Suppose by contradiction that there exist $\bar{x}_{1}, \bar{x}_{2} \epsilon$ $E_{w}(X, f)$ with $\bar{x}_{1} \neq \bar{x}_{2}$, satisfying $f\left(\bar{x}_{1}\right)=f\left(\bar{x}_{2}\right)=a$. On the other hand, since $a \in f\left(F_{w}(f, \omega)\right)$, there exists $\bar{x} \in F_{w}(f, \omega)$, satisfying $f(\bar{x})=a=\left(a_{1}, \ldots, a_{n}\right)$. Since $a \in f\left(F_{w}(f, w)\right)$ and $\bar{x}_{1}, \bar{x}_{2} \in E_{w}(X, f)$, we have

$$
\sum_{i=1}^{n} \omega_{i} f_{i}\left(\bar{x}_{1}\right)=\sum_{i=1}^{n} \omega_{i} f_{i}\left(\bar{x}_{2}\right)=\sum_{i=1}^{n} \omega_{i} a_{i} .
$$

It means that

$\bar{x}_{1}, \bar{x}_{2} \in F_{w}(f, \omega)=\left\{\bar{x} \in X: w_{f, \omega}(\bar{x})=\min _{x \in X} w_{f, \omega}(x)\right\}$.

Moreover, it follows from $\bar{x}_{1} \neq \bar{x}_{2}$ that there exists $\delta>0$, such that

$$
\overline{B_{\delta}\left(\bar{x}_{1}\right)} \cap \overline{B_{\delta}\left(\bar{x}_{2}\right)}=\varnothing .
$$

Define the mapping by

$$
\alpha(x)=\frac{\mathrm{d}\left(x, \overline{B_{\delta}\left(\overline{x_{2}}\right)}\right)}{\mathrm{d}\left(x, \overline{B_{\delta}\left(\overline{x_{1}}\right)}\right)+\mathrm{d}\left(x, \overline{B_{\delta}\left(\overline{x_{2}}\right)}\right)}, \quad x \in X .
$$

Let $\left\{\delta_{m}\right\}$ be a sequence of positive numbers satisfying $\delta_{m} \longrightarrow 0$. For every $m \in\{1,2, \ldots\}$, define the vector-valued function $f^{m}: X \longrightarrow \mathbb{R}^{n}$ by

$$
\begin{aligned}
& f_{i}^{m}(x)=f_{i}(x)-\alpha(x) \delta_{m}, \quad i=1, \ldots, n, \\
& f^{m}(x)=\left(f_{1}^{m}(x), \ldots, f_{n}^{m}(x)\right), \quad x \in X .
\end{aligned}
$$

Then, we obtain the vector-valued function sequence $\left\{f^{m}\right\}$; it is easy to check $\left\{f^{m}\right\} \subset C^{n}(X)$.

Since

$$
\begin{aligned}
\left\|f^{m}-f\right\|_{\infty} & =\max _{x \in X} \max \left\{\left|f_{1}^{m}(x)-f_{1}(x)\right|, \ldots,\left|f_{n}^{m}(x)-f_{n}(x)\right|\right\}, \\
& =\max _{x \in X} \max \left\{\left|\alpha(x) \delta_{m}\right|, \ldots,\left|\alpha(x) \delta_{m}\right|\right\}=\delta_{m} \max _{x \in X} \alpha(x) \leq \delta_{m} \longrightarrow 0,
\end{aligned}
$$

then $f^{m} \longrightarrow f$.

Take $\omega^{m} \longrightarrow \omega$; as $F_{\omega}$ is continuous at $(f, \omega)$ and $\left(f, \omega^{m}\right) \longrightarrow(f, \omega)$, there exists $x^{m} \in F_{w}\left(f, \omega^{m}\right)$, such that $x^{m} \longrightarrow \bar{x}_{1}$. From $x^{m} \in F_{w}\left(f, \omega^{m}\right)$, we have

$$
w_{f, \omega^{m}}\left(x^{m}\right)=\sum_{i=1}^{n} \omega_{i}^{m} f_{i}\left(x^{m}\right)=\min _{x \in X} \sum_{i=1}^{n} \omega_{i}^{m} f_{i}(x) .
$$

Since $x^{m} \longrightarrow \bar{x}_{1}$, there exists $N>0$, such that $x^{m} \in$ $B_{\delta}\left(\bar{x}_{1}\right)$ for all $m>N$. Next, we prove that when $m>N$, $F_{w}\left(f^{m}, \omega^{m}\right) \cap B_{\delta}\left(\overline{x_{2}}\right)=\varnothing$. If it is not true, then for some $m>N$, there exists $x_{2} \in B_{\delta}\left(\bar{x}_{2}\right)$ satisfying $x_{2} \in F_{w}\left(f^{m}, \omega^{m}\right)$, that is,

$$
\sum_{i=1}^{n} \omega_{i}^{m} f_{i}^{m}\left(x_{2}\right)=\min _{x \in X} \sum_{i=1}^{n} \omega_{i}^{m} f_{i}^{m}(x) \leq \sum_{i=1}^{n} \omega_{i}^{m} f_{i}^{m}\left(x^{m}\right) .
$$

Note that

$$
f_{i}^{m}\left(x_{2}\right)=f_{i}\left(x_{2}\right)+\alpha\left(x_{2}\right) \delta_{m}=f_{i}\left(x_{2}\right), \quad i=1, \ldots, n .
$$

Then,

$$
\begin{gathered}
\sum_{i=1}^{N} \omega_{i}^{m} f_{i}\left(x_{2}\right)=\sum_{i=1}^{n} \omega_{i}^{m} f_{i}^{m}\left(x_{2}\right) \leq \sum_{i=1}^{n} \omega_{i}^{m} f_{i}^{m}\left(x^{m}\right) \\
=\sum_{i=1}^{n} \omega_{i}^{m}\left[f_{i}\left(x^{m}\right)-\delta_{m}\right],=\sum_{i=1}^{n} \omega_{i}^{m} f_{i}\left(x^{m}\right)-\delta_{m} .
\end{gathered}
$$

Hence,

$$
\sum_{i=1}^{n} \omega_{i}^{m} f_{i}\left(x_{2}\right)<\sum_{i=1}^{n} \omega_{i}^{m} f_{i}\left(x^{m}\right) .
$$

It is in contradiction with $\sum_{i=1}^{n} \omega_{i}^{m} f_{i}\left(x^{m}\right)=$ $\min \sum_{i=1}^{n} \omega_{i}^{m} f_{i}(x)$.

$x \in X$ Hence, when $m>N, F_{w}\left(f^{m}, \omega^{m}\right) \cap B_{\delta}\left(\overline{x_{2}}\right)=\varnothing$, it is in contradiction with the continuity of $F_{\omega}$ at $(f, \omega)$. Therefore, $a$ corresponds to the unique Pareto weakly efficient solution.

Based on Theorem 1, we obtain a result about pointwise Tykhonov well-posedness in vector optimization problems as below.

Definition 5. The vector optimization problem (2) is called Tykhonov well-posed at $a \in f\left(E_{w}(X, f)\right)$, if there exists a unique $\bar{x}$ in $E_{w}(X, f)$, such that $f(\bar{x})=a$; then, for any $\left\{x_{m}\right\} \subset X$ satisfying $f\left(x_{m}\right) \longrightarrow a$, we have that $x_{m} \longrightarrow \bar{x}$.

Remark 2. If the vector optimization problem (2) is a scalar optimization, then Definition 5 is Tykhonov well-posed for the scalar case. 
Theorem 3. There exists a dense residual subset $Q \subset C^{n}(X) \times \mathbb{R}^{n}$, such that for every $(f, a) \in Q$, if $a \in f\left(E_{w}(X, f)\right)$, the vector optimization problem (2) is Tykhonov well-posedness at a.

Proof. By Theorem 1, there exists a dense residual subset $Q \subset C^{n}(X) \times \mathbb{R}^{n}$, such that for every $(f, a) \in Q$, if $a \in f\left(E_{w}(X, f)\right)$, then the vector a corresponds to a unique Pareto weakly efficient solution of $V(f, a)$. As $a \in f\left(E_{w}(X, f)\right)$, there exist some $\bar{x} \in E_{w}(X, f)$, such that $f(\bar{x})=a$. Assume that $\left\{x_{m}\right\} \subset X$ with $f\left(x_{m}\right) \longrightarrow a$; since $X$ is compact, $\left\{x_{m}\right\}$ has a convergent subsequence $\left\{x_{m_{k}}\right\}$, and let $x_{m_{k}} \longrightarrow \hat{x}$. Since $f^{-1}(a)=\{\bar{x}\}$ and $f\left(x_{m}\right) \longrightarrow a$, it is easy to see that for all subsequences of $\left\{x_{m}\right\}$ converging to $\hat{x}$, we have that $x_{m} \longrightarrow \bar{x}$. Therefore, the vector optimization problem (2) is Tykhonov wellposedness at $a$.

\section{Conclusion}

In this paper, the authors solve the problems mentioned in the introduction. By use of nonlinear scalarization method, in the sense of Baire category, for most points $(f, a)$ constituting a dense residual subset $Q$ of the product space $C^{n}(X) \times \mathbb{R}^{n}$, if the vector $a$ is a Pareto weakly efficient point of the function $f$, then $f^{-1}(a)$ is the unique Pareto weakly efficient solution of vector optimization problem $V(X, f)$ corresponding to $a$. Obviously, if the vector $a$ is a Pareto-efficient point of the function $f$, the result still holds. As applications of the uniqueness, in the sense of Baire category, for most $(f, a)$, if the vector $a$ is a Pareto weakly efficient point, the authors show that vector optimization problem (2) is Tykhonov well-posed at the vector $a$. By means of the weighting method which is a linear scalarization method, similar uniqueness result is obtained.

\section{Data Availability}

No data were used to support this study.

\section{Conflicts of Interest}

The authors declare that there are no conflicts of interest regarding the publication of this paper.

\section{Acknowledgments}

This study was supported by the National Natural Science Foundation of China (grant nos. 11861025, 11761023, and 71961003), the Doctoral Program Fund of Ministry of Education of the People's Republic of China (20115201110002), the Program for the Outstanding Science and Technology Innovative Talents of Higher Education of Guizhou (Qian Jiao He KY Zhi 2017[081]), and the Natural Science Foundation of Guizhou Province ([2018]5769, [2017]5788, and LH [2017]7223).

\section{References}

[1] P. S. Kenderov, "Most of the optimization problems have unique solution," Comptes Rendus de Academic Bulgare Sciences, vol. 37, pp. 297-299, 1984.

[2] G. Beer, "On a generic optimization theorem of Petar Kenderov," Nonlinear Analysis: Theory, Methods \& Applications, vol. 12, no. 6, pp. 647-655, 1988.

[3] V. L. Levin, "On generic uniqueness of optimal solutions for the general Monge-Kantorovich problem," Set-Valued Analysis, vol. 9, no. 4, pp. 383-390, 2001.

[4] V. L. Levin, "On generic uniqueness of optimal solution in an infinite dimensional linear programming problem," Doklady Mathematics, vol. 78, no. 1, pp. 490-492, 2008.

[5] M. A. Goberna, M. A. Lopez, and M. I. Todorov, "A generic result in linear semi-infinite optimization," Applied Mathematics Optimization, vol. 48, pp. 181-193, 2003.

[6] N. G. Persico, "Generic uniqueness of the solutions to a continuous linear programming problem," SSRN Electronic Journal, vol. 5-10, p. 6, 2005.

[7] A. Ioffe and R. E. Lucchetti, "Generic well-posedness in minimization problems," Abstract and Applied Analysis, vol. 2005, no. 4, pp. 343-360, 2005.

[8] A. J. Zaslavski, "Generic well-posedness of minimization problems with mixed continuous constraints," Nonlinear Analysis: Theory, Methods \& Applications, vol. 64, no. 11, pp. 2381-2399, 2006.

[9] P. S. Kenderov and N. K. Ribarska, "Most of the two person zero-sum games have unique solution," in Proceedings of the Workshop Mini-Conference on Functional Analysis and Optimization, vol. 20, pp. 73-82, Canberra, Australia, August 1988.

[10] K. K. Tan, J. Yu, and X. Z. Yuan, "The uniqueness of saddle points," Bulletin of the Polish Academy of Sciences Mathematics, vol. 43, pp. 119-129, 1995.

[11] J. Yu, D. Peng, and S. Xiang, "Generic uniqueness of equilibrium points," Nonlinear Analysis: Theory, Methods \& Applications, vol. 74, no. 17, pp. 6326-6332, 2011.

[12] D. T. Peng, J. Yu, and N. H. Xiu, "Generic uniqueness theorems with some applications," Journal of Global Optimization, vol. 56, pp. 712-725, 2013.

[13] B. Cao, S. Fan, J. Zhao, P. Yang, K. Muhammad, and M. Tanveer, "Quantum-enhanced multiobjective large-scale optimization via parallelism," Swarm and Evolutionary Computation, vol. 57, Article ID 100697, 2020.

[14] B. Cao, J. Zhao, Y. Gu, Y. Ling, and X. Ma, “Applying graphbased differential grouping for multiobjective large-scale optimization," Swarm and Evolutionary Computation, vol. 53, Article ID 100626, 2020.

[15] B. Cao, W. Dong, Z. Lv, Y. Gu, S. Singh, and P. Kumar, "Hybrid microgrid many-objective sizing optimization with fuzzy decision," IEEE Transactions on Fuzzy Systems, vol. 28, no. 11, pp. 2702-2710, 2020.

[16] A. N. Tykhonov, "On the stability of the functional optimization problem," USSR Computational Mathematics and Mathematical Physics, vol. 6, no. 4, pp. 28-33, 1966.

[17] A. L. Dontchev and T. Zolezzi, Well-posed Optimization Problems, Springer, Berlin, Germany, 1993.

[18] R. Lucchetti, "Well posedness, towards vector optimization," Recent Advances and Historical Development of Vector Optimization Problems, vol. 294, 1987.

[19] P. Loridan, "Well posedness in vector optimization," Recent Developments in Well Posed-Variational Problems, vol. 331, 1995. 
[20] X. X. Huang, "Extended well-posedness properties of vector optimization problems," Journal of Optimization Theory and Applications, vol. 106, no. 1, pp. 165-182, 2000.

[21] X. X. Huang, "Pointwise well-posedness of perturbed vector optimization problems in a vector-valued variational principle," Journal of Optimization Theory and Applications, vol. 108, no. 3, pp. 671-684, 2001.

[22] E. Miglierina, E. Molho, and M. Rocca, "Well-posedness and scalarization in vector optimization," Journal of Optimization Theory and Applications, vol. 126, no. 2, pp. 391-409, 2005.

[23] G. P. Crespi, M. Papalia, and M. Rocca, "Extended wellposedness of quasiconvex vector optimization problems," Journal of Optimization Theory and Applications, vol. 141, no. 2, pp. 285-297, 2009.

[24] A. J. Zaslavski, Optimization on Metric and Normed Spaces, Springer, New York, NY, USA, 2010.

[25] S. Kapoor and C. S. Lalitha, "Stability and scalarization for a unified vector optimization problem," Journal of Optimization Theory and Applications, vol. 182, no. 3, pp. 1050-1067, 2019.

[26] T. Zolezzi, "Extended well-posedness of optimization problems," Journal of Optimization Theory and Applications, vol. 91, no. 1, pp. 257-266, 1996.

[27] H. W. Corley, "An existence result for maximizations with respect to cones," Journal of Optimization Theory and Applications, vol. 31, no. 2, pp. 277-281, 1980.

[28] G. Y. Chen, X. X. Huang, and X. Q. Yang, Vector Optimization: Set-Valued and Variational Analysis, Springer, Berlin, Germany, 2005.

[29] C. Gerstewitz, "Nichtkonvexe dualit $\ddot{a} \mathrm{t}$ in der Vektoroptimierung," Wissensch, Zeitschr, 1983.

[30] D. T. Luc, Theory of Vector Optimization, Springer, Berlin, Germany, 1989.

[31] J. Yu, "Essential weak efficient solution in multiobjective optimization problems," Journal of Mathematical Analysis and Applications, vol. 166, pp. 230-235, 1992.

[32] S. W. Xiang and W. S. Yin, "Stability results for efficient solutions of vector optimization problems," Journal of Optimization Theory and Applications, vol. 134, no. 3, pp. 385398, 2007. 